\title{
High Demand: Identification of Dermatology Visit Trends from 1991-2016 National Ambulatory Medical Care Surveys
}

Jade Conway, BA ${ }^{1}$, Bipasha Roy, BA ${ }^{1}$, Lauren Barazani, $\mathrm{MS}^{1}$, Albert G. Wu, MS ${ }^{1}$, Abigail Cline, $\mathrm{PhD}, \mathrm{MD}^{2}$, Janet Moy, $\mathrm{MD}^{2}$

${ }^{1}$ New York Medical College School of Medicine, Valhalla, NY

${ }^{2}$ Department of Dermatology, Metropolitan Hospital, New York, NY

\section{ABSTRACT}

Background: The field of dermatology is constantly evolving and expanding to accommodate for increased demand. In order to maximize future productivity, it is important to recognize and understand how the desires of patients and the nature of physician visits have changed over time.

Objective: To evaluate and provide evidence-based reasoning for the changes occurring in the field of dermatology.

Methods: Analysis of the 1991-2016 National Summary Tables from the National Ambulatory Medical Care Survey (NAMCS) was performed in order to identify several trends relating to dermatology visits.

Results: Annual visit rates to dermatologists have increased by $68 \%$, while visit length has increased by $39 \%$. Drug visits have increased by $86 \%$, while drug mentions increased by $370.2 \%$.

Limitations: Limitations of this paper include a limited narrow timeline for data points.

Conclusion: As the field of dermatology will continue to expand in the future, dermatologists can expect to be busier than ever before. We expect that more patients will seek care, physician visits will be longer, and chief complaints and treatment options will continue to expand and vary.

\section{INTRODUCTION}

As demand for dermatology grows, physicians contend with more treatment options, electronic medical records, and billing systems. Over a third of dermatologists report burnout, citing longer hours, excessive documentation, and lack of protected time. ${ }^{1}$ To maximize productivity and anticipate this field's evolution, we should understand how dermatology has changed. Utilizing the National Summary
Tables from the National Ambulatory Medical Care Survey from 1991-2016, we analyzed office data and identified trends relating to dermatology visits over time.

\section{RESULTS}

From 1991 to 2016, annual visit rates to dermatologists increased 68\% (Figure 1). Visit length, defined as the time a patient spends with their dermatologist, increased $39 \%$. In 1991, the average dermatology visit 
Figure 1: Annual rate of dermatology visits.

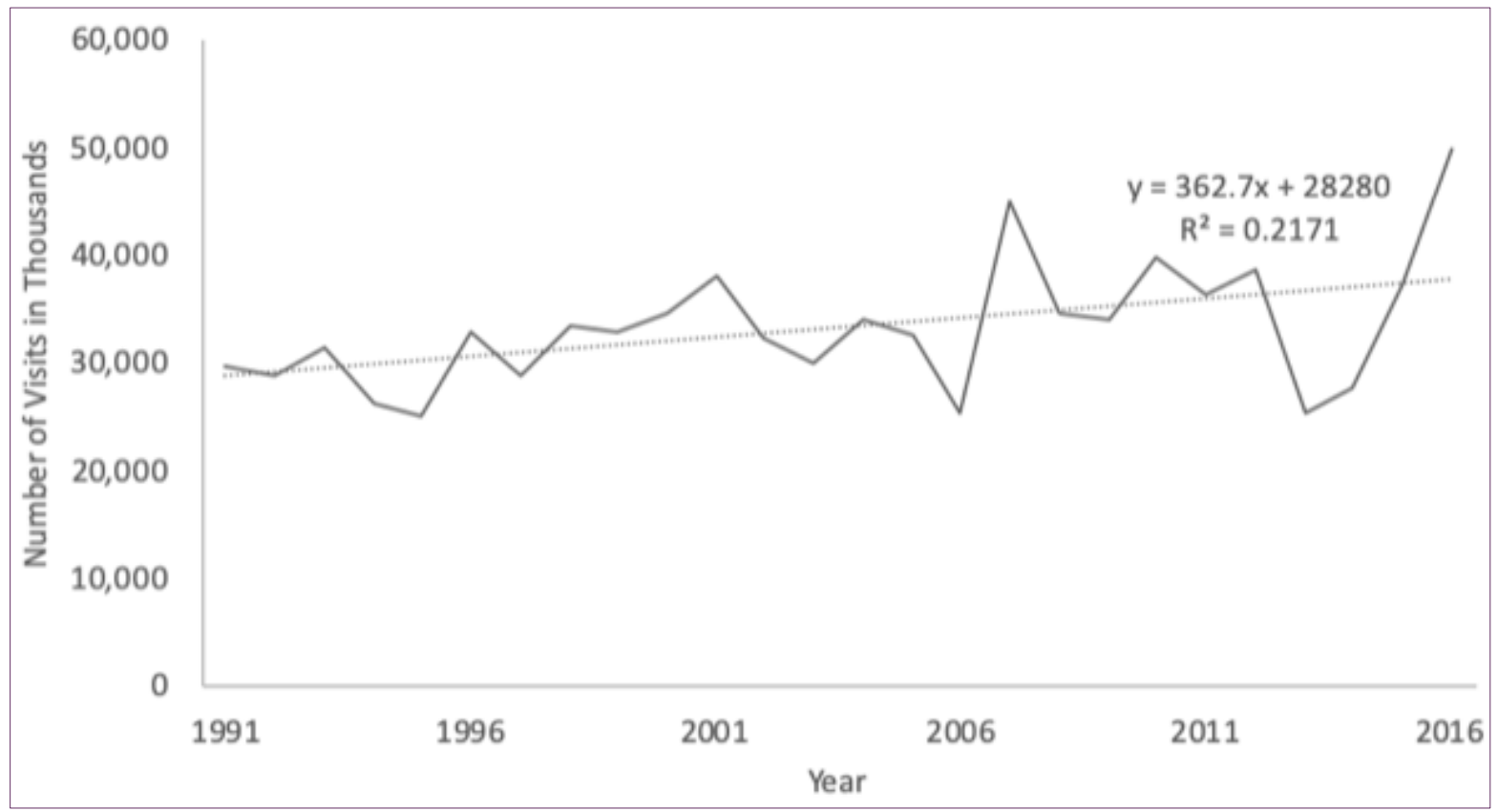

Figure 2. Annual rate of drug visits (visits at which one or more drugs were provided or prescribed) and drug mentions (documentation in a patient's record of a drug provided, prescribed, or continued at a visit).

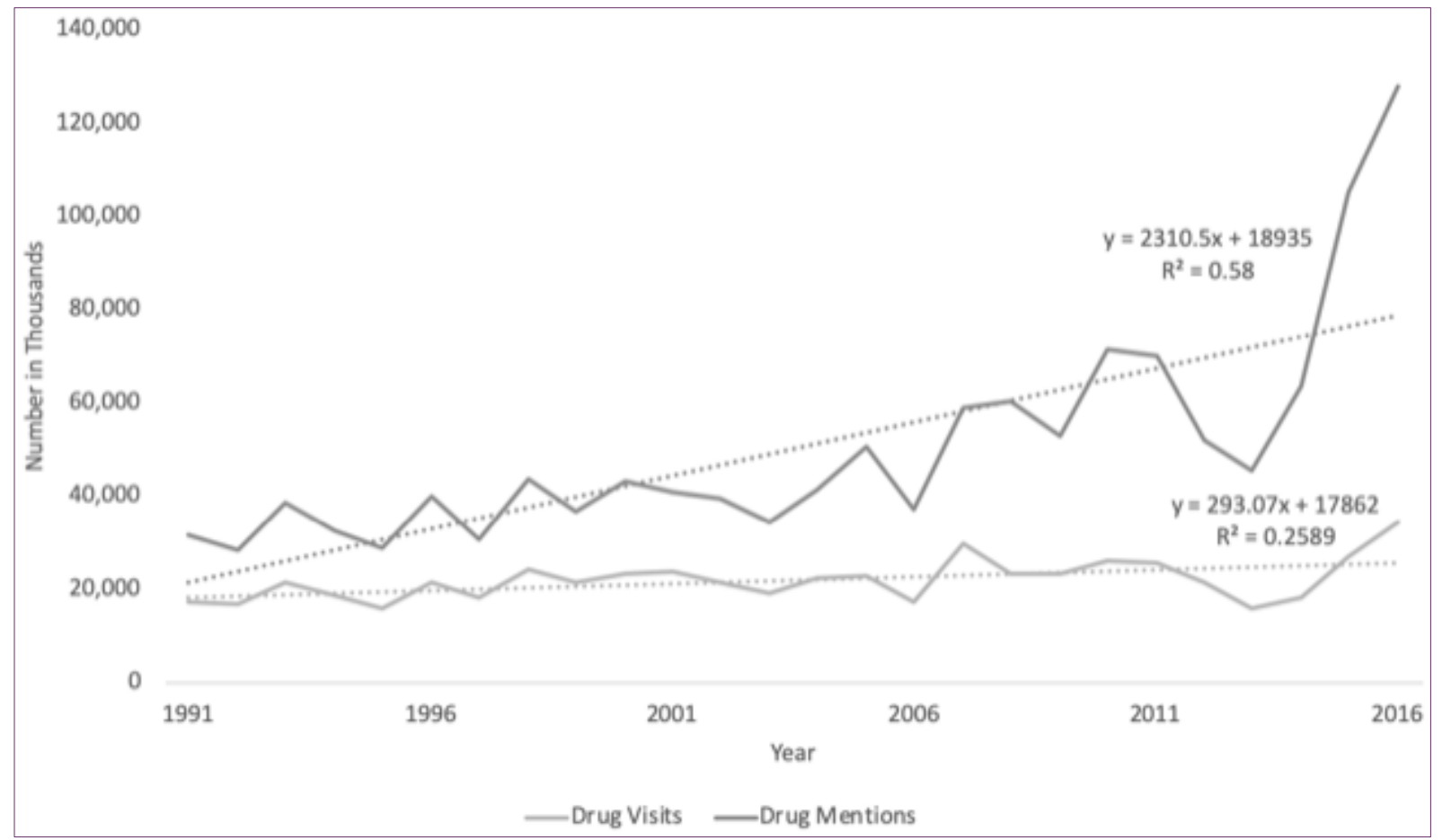

November 2020 Volume 4 Issue 6 
length was 14.9 minutes. In 2016, the average length was 20.7 minutes. The greatest change comes from the number of drug visits and mentions over time. A drug visit is an encounter where one or more drugs are prescribed or provided by the physician, while a drug mention is where a drug being prescribed, provided, or continued was documented in the patient's record; drug mentions include all the drug visits. $^{2}$ From 1991 to 2016, drug visits increased $86 \%$, while drug mentions increased $370.2 \%$ (Figure 2). The percent of visits with drug mentions increased by $21 \%$, from $57.2 \%$ in 1991 to $69.1 \%$ in 2016 .

Dermatologists now offer treatments including medical interventions to aesthetic procedures. This expansion may compel more patients to seek consultations, thereby increasing the numbers of annual visits. Dermatologists spend more hours in the office each year, increasing availability for patients to secure appointments. ${ }^{1}$ The increased incidence of nonmelanoma and melanoma skin cancers may also account for the increased visits, as the average annual number of adults treated for skin cancer increased from 3.4 million in 20022006 to 4.9 million in 20072011.3 Additionally, an increase in drug visits results in increased follow-up visits, as many dermatological conditions require lifelong management.

Social media may also play a role in the increase in patient visits. With readily available information on the internet, patients are more aware of their conditions and treatment options. Another influence may be social media advertisements for cosmetic procedures, as well as posts of other patients' procedural experiences. After viewing an online photograph highlighting a dissatisfying feature, over $80 \%$ of patients sought out aesthetic correction. ${ }^{4}$

\section{DISCUSSION}

Explanations for longer dermatological visits include more patient concerns, discussion of treatment plan, and insurance requirements. The increase in dermatological procedures may be due to evolving beauty trends as well as greater physician interest in performing cosmetic procedures. ${ }^{5}$ Surgical and cosmetic procedures can be time consuming, thus contributing to the increased visit length.

Steps taken in recent years to address these changes include increased utilization of support staff, physician extenders, and telemedicine. ${ }^{6}$ The percentage of dermatologists in solo practice dropped from $44 \%$ in 2005 to $35 \%$ in 2014 . Employment of physician assistants or nurse practitioners increased from $28 \%$ in 2005 to $46 \%$ in $2014 .{ }^{6}$ Working in a group-based practice may allow physicians to spend less time performing administrative duties and more time seeing patients. Teledermatology is another cost-effective strategy to decrease patient wait times while increasing satisfaction and access to healthcare.

\section{CONCLUSION}

Dermatology remains a growing field, pushed to evolve by advances in technology and healthcare demand. Looking at the change in dermatology visits through the decades, we anticipate that visits will become longer, more patients will seek care, and dermatologists may be busier than ever before. Further research is required to better understand the trajectory of the field, as the trends we identify are extrapolated from a narrow timeline of data points. 


\section{Conflict of Interest Disclosures: None}

Funding: None

\section{Corresponding Author:}

Jade Conway, BA

New York Medical College School of Medicine

40 Sunshine Cottage Rd

Valhalla, NY 10595, United States

Email: jconway2@student.nymc.edu

\section{References:}

1. Colon A, Gillihan R, Motaparthi K. Factors contributing to burnout in dermatologists. Clin Dermatol. 2020; 38(3): 321-327. doi:10.1016/j.clindermatol.2020.02.002

2. NAMCS/NHAMCS Ambulatory Health Care Data Homepage. Centers for Disease Control and Prevention. Available from: https://www.cdc.gov/nchs/ahcd/.

3. Guy GP Jr, Machlin SR, Ekwueme DU, Yabroff $\mathrm{KR}$. Prevalence and costs of skin cancer treatment in the U.S., 2002-2006 and 2007-2011. Am J Prev Med. 2015;48(2):183-187. doi:10.1016/j.amepre.2014.08.036

4. Ross NA, Todd Q, Saedi N. Patient seeking behaviors and online personas: social media's role in cosmetic dermatology. Dermatol Surg. 2015;41(2):269-276. doi:10.1097/DSS.0000000000000267

5. Neville JA, Housman TS, Letsinger JA, Fleischer $A B$ Jr, Feldman SR, Williford PM. Increase in procedures performed at dermatology office visits from 1995 to 2001. Dermatol Surg. 2005;31(2):160-162. doi:10.1111/j.15244725.2005.31037

6. Ehrlich A, Kostecki J, Olkaba H. Trends in dermatology practices and the implications for the workforce. J Am Acad Dermatol. 2017;77(4):746-752. doi:10.1016/j.jaad.2017.06.030 